\title{
PSR J1618-3921: a recycled pulsar in an eccentric orbit
}

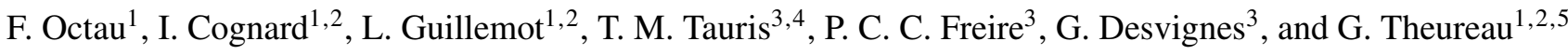 \\ ${ }^{1}$ Laboratoire de Physique et Chimie de l'Environnement et de l'Espace - Université d'Orléans/CNRS, 45071 Orléans Cedex 02, \\ France \\ e-mail: franck.octau@cnrs-orleans.fr \\ 2 Station de radioastronomie de Nançay, Observatoire de Paris, CNRS/INSU, 18330 Nançay, France \\ ${ }^{3}$ Max-Planck-Institut für Radioastronomie, Auf dem Hügel 69, 53121 Bonn, Germany \\ ${ }^{4}$ Argelander-Institut für Astronomie, Universität Bonn, Auf dem Hügel 71, 53121 Bonn, Germany \\ ${ }^{5}$ Laboratoire Univers et Théories (LUTh), Observatoire de Paris, CNRS/INSU, Université Paris Diderot, 5 place Jules Janssen, \\ 92190 Meudon, France
}

Received 11 September 2017 / Accepted 21 January 2018

\begin{abstract}
Context. The 11.99 ms pulsar PSR J1618-3921 orbits a He white dwarf companion of probably low mass with a period of $22.7 \mathrm{~d}$. The pulsar was discovered in a survey of the intermediate Galactic latitudes at $1400 \mathrm{MHz}$ that was conducted with the Parkes radio telescope in the late 1990s. Although PSR J1618-3921 was discovered more than 15 years ago, only limited information has been published about this pulsar, which has a surprisingly high orbital eccentricity $(e \simeq 0.027)$ considering its high spin frequency and the likely low mass of the companion.

Aims. The focus of this work is a precise measurement of the spin and the astrometric and orbital characteristics of PSR J1618-3921. This was done with timing observations made at the Nançay Radio Telescope from 2009 to 2017.

Methods. We analyzed the timing data recorded at the Nançay Radio Telescope over several years to characterize the properties of PSR J1618-3921. A rotation ephemeris for this pulsar was obtained by analyzing the arrival times of the radio pulses at the telescope. Results. We confirm the unusual eccentricity of PSR J1618-3921 and discuss several hypotheses regarding its formation in the context of other discoveries of recycled pulsars in eccentric orbits.
\end{abstract}

Key words. pulsars: individual: PSR J1618-3921 - ephemerides

\section{Introduction}

The pulsar PSR J1618-3921 is a Galactic-disk recycled pulsar discovered in a $1.4-\mathrm{GHz}$ survey of the intermediate Galactic latitudes with the Parkes radio telescope (Edwards \& Bailes 2001). It is in a binary system with a likely low-mass He white dwarf (WD) companion; the orbit has a period of 22.7 days. In the "standard" pulsar recycling scenario, millisecond pulsars (MSPs, here defined as pulsars with spin periods shorter than $15 \mathrm{~ms}$ ) are spun-up by mass and angular momentum transfer from a companion star in a low-mass X-ray binary (Alpar et al. 1982; Radhakrishnan \& Srinivasan 1982; Bhattacharya \& van den Heuvel 1991; Tauris \& van den Heuvel 2006). This process leads to highly circularized orbits, that is, orbits with very low eccentricities, $e$ (Phinney \& Kulkarni 1994). Early pulsar timing observations of PSR J1618-3921, however, revealed an anomalously high eccentricity of about 0.027 (Bailes 2010). This pulsar has thus been classified as an eccentric millisecond pulsar (eMSP, defined here as MSPs with $e>0.01$ ), an emerging subclass of MSPs comprising a handful of objects (see Table 1 and references therein). Interestingly, PSR J1618-3921 and the other MSPs in this growing pulsar class display very similar orbital periods and eccentricity values (see Table 1), which suggests a common, non-chaotic formation process (Freire \& Tauris 2014; Antoniadis 2014; Jiang et al. 2015). These formation processes and the orbital parameters for PSR J1618-3921 are discussed in detail in Sect. 3.

Although several of the above-listed publications mentioned PSR J1618-3921, a complete description of the timing properties and orbital characteristics of this pulsar has been lacking since its discovery more than a decade ago. We here present the results of the long-term timing of PSR J1618-3921 with the Nançay decimetric Radio Telescope (NRT), confirming the unusual eccentricity of the pulsar. The timing analysis is presented in Sect. 2. In Sect. 3 we discuss the main results together with different models that have been proposed to explain the formation of PSR J1618-3921 and other eMSPs.

\section{Observations and data analysis}

Observations of PSR J1618-3921 with the NRT at $1.4 \mathrm{GHz}$ began in May 2009. First observations were conducted with the Berkeley - Orléans - Nançay (BON) instrumentation, with very limited information regarding the pulsar spin parameters, orbital parameters, and dispersion measure (DM). We therefore carried out observations with the BON backend in so-called "survey" mode, that is, with a $512 \times 0.25 \mathrm{MHz}$ incoherent filter bank sampled every $32 \mu \mathrm{s}$, giving us enough flexibility to search for the pulsar parameters in each observation and find its pulsations. After August 2011, pulsar observations at the NRT used the NUPPI instrumentation, a version of the Green Bank Ultimate Pulsar Processing Instrument ${ }^{1}$ (GUPPI) designed for Nançay. At first, observations of PSR J1618-3921 with NUPPI were also conducted in survey mode, with a total bandwidth of $512 \mathrm{MHz}$ divided in 1024 channels of $0.5 \mathrm{MHz}$ sampled every $64 \mu$ s. Survey-mode NUPPI observations were conducted until a robust timing solution for PSR J1618-3921 became available

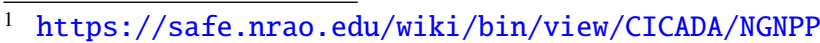


Table 1. Rotational period, orbital period, companion mass, orbital eccentricity, and height above the Galactic plane for the six known eMSPs.

\begin{tabular}{ccccccc}
\hline \hline PSR & $P(\mathrm{~ms})$ & $P_{b}$ (days) & $m_{c}\left(M_{\odot}\right)$ & $e$ & $|z|(\mathrm{kpc})$ & References \\
\hline $\mathrm{J} 0955-6150$ & 2.00 & 24.6 & 0.22 & 0.11 & 0.22 & Camilo et al. (2015) \\
$\mathrm{J} 1618-3921$ & 11.99 & 22.7 & 0.20 & 0.027 & 0.75 & Edwards \& Bailes (2001), Bailes (2010) \\
$\mathrm{J} 1946+3417$ & 3.17 & 27.0 & $0.2556(19)$ & 0.14 & 0.57 & Barr et al. (2013, 2017) \\
$\mathrm{J} 1950+2414$ & 4.30 & 22.2 & 0.30 & 0.08 & 0.15 & Knispel et al. (2015) \\
$\mathrm{J} 2234+0611$ & 3.58 & 32.0 & 0.23 & 0.13 & 0.60 & Deneva et al. (2013), Antoniadis et al. (2016) \\
\hline $\mathrm{J} 1903+0327$ & 2.15 & 95.2 & $1.029(8)$ & 0.44 & 0.1 & Champion et al. (2008), Freire et al. (2011) \\
\hline
\end{tabular}

Notes. Unlike other pulsars in this table, PSR J1903+0327 is believed to have been formed by triple-star interaction and is therefore isolated in the list. The quoted masses are the median masses derived from the mass function assuming a pulsar mass of $1.35 M_{\odot}$ and a random distribution of orbital inclinations, except for PSR J1903+0327 and PSR J1946+3417, for which the pulsar and companion masses were determined through the measurement of post-Keplerian parameters. The height above the Galactic plane, $z$, was obtained from the ATNF pulsar catalog (see http://www.atnf.csiro.au/research/pulsar/psrcat, Manchester et al. 2005). The $z$ values all assume the YMW16 model of Yao et al. (2017).

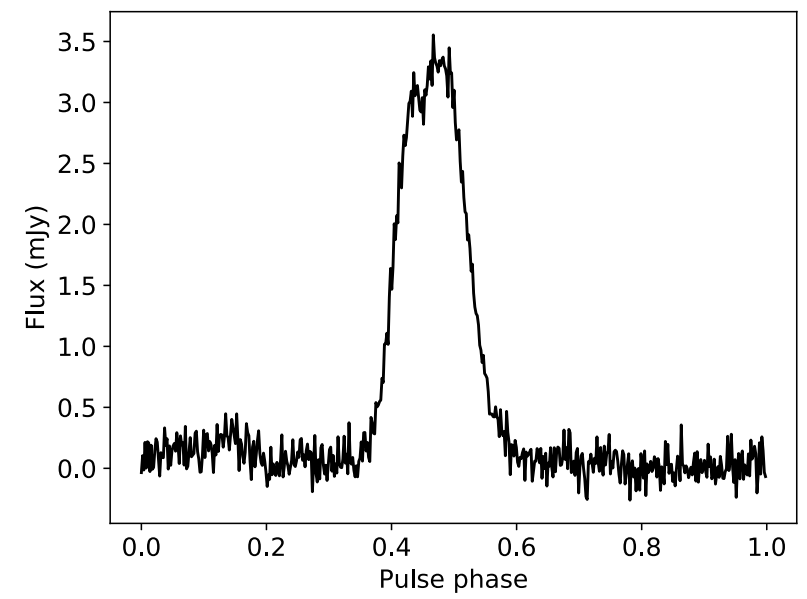

Fig. 1. Pulse profile for PSR J1618-3921 at $1.4 \mathrm{GHz}$ obtained by integrating $40 \mathrm{~h}$ of NRT observations conducted with the NUPPI backend in timing mode between MJDs 56998 and 57869. The initial 2048 phase bins were divided by four in this graph to improve readability.

and enabled us to observe the pulsar in the regular "timing" mode, which we did starting from December 2014. In the regular timing mode of NUPPI, 128 channels of $4 \mathrm{MHz}$ are coherently dedispersed in real time and the time series are folded online. In total, 10 survey mode observations of PSR J1618-3921 were made with the BON instrumentation, and 60 were conducted with the NUPPI pulsar backend, including the first 8 made in survey mode. Table 2 summarizes our observations of the pulsar, and Fig. 1 displays a pulse profile for PSR J1618-3921, obtained by summing all timing-mode NUPPI observations. The profile comprises one main broad peak showing substructures, and evidence for a secondary peak leading the main one by approximately 0.35 rotation.

The data were reduced using the PSRCHIVE software library (Hotan et al. 2004). The data were cleaned of radio frequency interference (RFI) using a median-smoothed automatic channel-zapping algorithm. The polarization information was calibrated with the SINGLEAXIS method of PSRCHIVE. For each observation configuration (see Table 2), we combined all available $1.4 \mathrm{GHz}$ observations and smoothed the resulting integrated profiles to form template profiles. The summed timing-mode NUPPI profile at $1.4 \mathrm{GHz}$ before smoothing is shown in Fig. 1. Finally, times of arrival (TOAs) were created by cross-correlating time- and frequency-scrunched observations with the template profiles. We obtained a dataset of 70 TOAs. The main properties of these TOAs are given in Table 2 .

The analysis of the TOA data was conducted with TEMPO2 (Hobbs et al. 2006). Because of the low signal-to-noise ratio $(\mathrm{S} / \mathrm{N})$ of the pulse profiles, in particular for the survey mode observations, we used fully frequency-scrunched TOAs in our timing analysis. Therefore, we could not determine the DM jointly with the other timing parameters. Nevertheless, in a first step of our analysis, we split timing-mode NUPPI observations into four sub-bands of $128 \mathrm{MHz}$, and analyzed the TOAs with TEMPO2, fitting for the DM and all other rotation parameters. We measured DM $=117.965 \pm 0.011 \mathrm{pc} \mathrm{cm}^{-3}$. In a last analysis step, we fixed the DM at the latter value and analyzed the fully time- and frequency-scrunched TOAs. We fit systematic time offsets between the different TOA datasets (i.e., BON survey, NUPPI survey, and NUPPI timing) to account for differences in template profiles and observing systems and configurations. In addition, we fit for the sky position, rotational period, and first time derivative of the pulsar, as well as for the five Keplerian parameters describing the orbit. The TOA uncertainties were weighted using TEMPO2 EFAC factors (Hobbs et al. 2006) to account for potential under- or overestimations of the errors. Different EFAC factors were used for each of the TOA datasets; their values are listed in Table 2 . The best-fit parameters from the timing analysis are listed in Table 3, and the differences between the measured TOAs and the predictions of our best-fit model, the so-called residuals, are plotted in Fig. 2 . The weighted residuals have an RMS of $\sim 25.5 \mu \mathrm{s}$, or about $2 \times 10^{-3}$ of the pulsar rotational period. The reduced $\chi^{2}$ of approximately 1.2 indicates an acceptable agreement between the best-fit model and the data.

With the measured projected semi-major axis of the orbit, $x$, and orbital period, $P_{b}$, we derived the mass function given by

$f\left(m_{\mathrm{p}}, m_{\mathrm{c}}\right)=\frac{\left(m_{\mathrm{c}} \sin \mathrm{i}\right)^{3}}{\left(m_{\mathrm{p}}+m_{\mathrm{c}}\right)^{2}}=\frac{4 \pi}{T_{\odot}} \frac{x^{3}}{P_{b}^{2}}=0.00225347(9) M_{\odot}$,

where $m_{\mathrm{p}}$ is the pulsar mass, $m_{\mathrm{c}}$ is the companion mass, $T_{\odot}=G M_{\odot} / c^{3}=4.925490947 \mu \mathrm{s}$, and $i$ is the orbital inclination angle. Assuming an edge-on orbit $\left(i=90^{\circ}\right)$ and a pulsar mass of $1.35 M_{\odot}$, we can determine a lower limit on the companion mass $m_{\mathrm{c}}$ of $0.17 M_{\odot}$. As noted in Lorimer \& Kramer (2004), the probability of observing a binary system with an inclination lower than $i_{0}$ for a random distribution of orbital inclinations is $1-\cos \left(i_{0}\right)$; therefore, a $90 \%$ confidence upper limit on the companion mass can be derived by assuming an inclination angle $i$ of $26^{\circ}$. This gives an upper limit of $0.44 M_{\odot}$ for 
Table 2. Summary of observations of PSR J1618-3921 with the NRT.

\begin{tabular}{lccc}
\hline \hline Instrumentation & BON & NUPPI & NUPPI \\
\hline Observation mode & Survey & Survey & Timing \\
MJD range & $54963-55647$ & $56573-56946$ & $56998-57869$ \\
Calendar dates & May 2009-March 2011 & October 2013-October 2014 & December 2014-April 2017 \\
Number of observations & 10 & 8 & 52 \\
Total observation time & $6 \mathrm{~h} 56 \mathrm{~min}$ & $3 \mathrm{~h} 26 \mathrm{~min}$ & $41 \mathrm{~h} 16 \mathrm{~min}$ \\
Center frequency (MHz) & 1398 & 1484 & 1484 \\
Bandwidth (MHz) & 128 & 512 & 512 \\
Mean TOA uncertainty & $220 \mu \mathrm{s}$ & $193 \mu \mathrm{s}$ & $26 \mu \mathrm{s}$ \\
EFAC & 1.10 & 0.67 & 1.17 \\
\hline
\end{tabular}

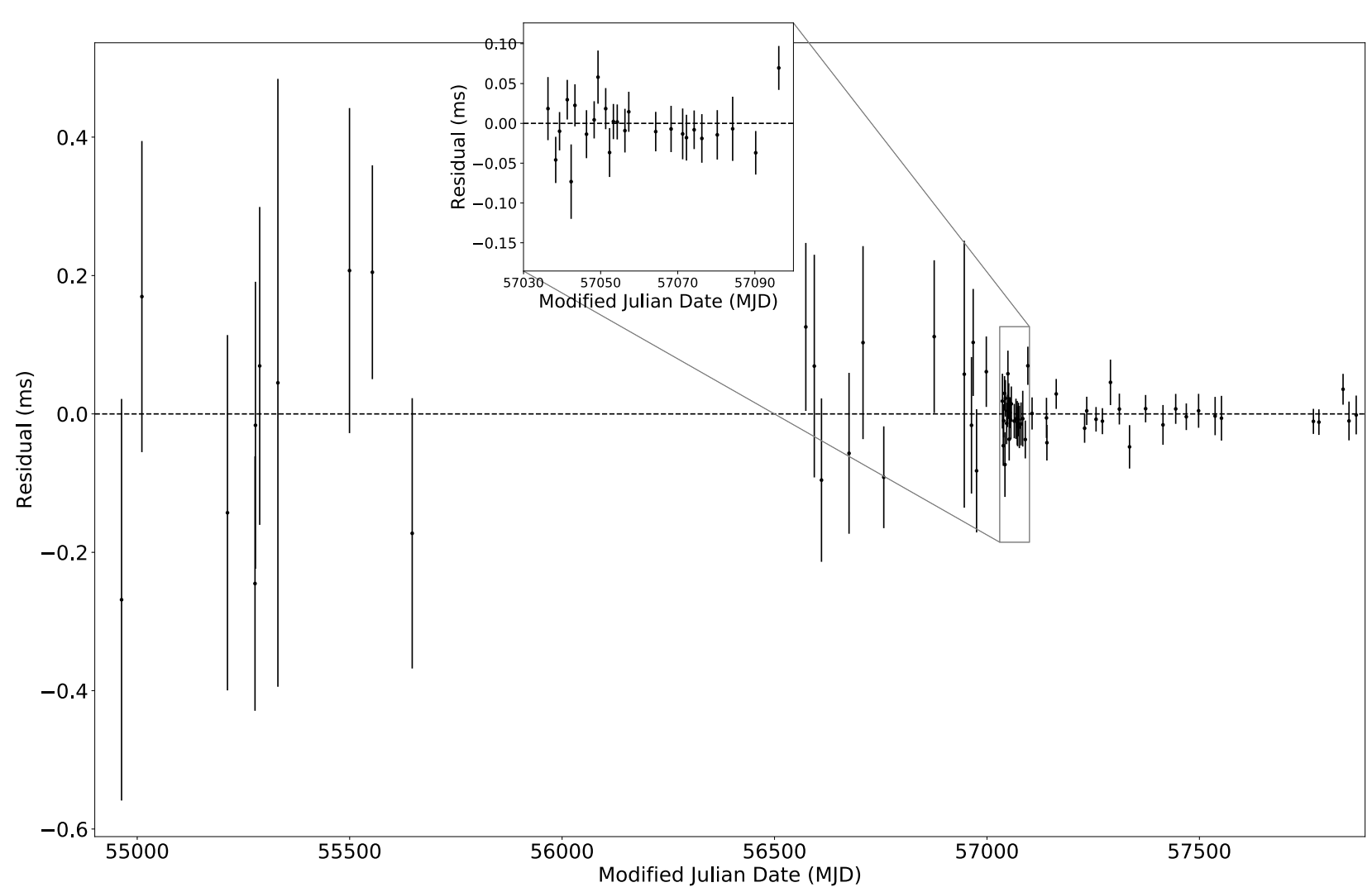

Fig. 2. Timing residuals as a function of time for the best-fit timing solution of PSR J1618-3921 given in Table 3. The inset shows the residuals for the time interval when the pulsar was intensively observed to derive a phase-connected timing solution.

the companion mass of PSR J1618-3921, with a median mass of $0.20 M_{\odot}$. The minimum mass of CO-type WDs is $0.33 M_{\odot}$ (e.g., Tauris et al. 2012). A mass like this is only possible if the inclination angle is smaller than $34^{\circ}$, which has a probability of 0.17 assuming a random orbital orientation. Furthermore, the median companion mass fits the $\left(M_{\mathrm{WD}}, P_{b}\right)$ correlation for He WDs (Tauris \& Savonije 1999) quite well. The value of the mass function and the range of likely companion mass values, combined with the orbital period, therefore indicate that the companion star is most likely a He-type WD. The orbital eccentricity $e$ of $0.0274339(10)$ is in line with the value reported by Bailes (2010), confirming the unusually high orbital eccentricity of PSR J1618-3921, in spite of its common orbital period and companion type. Considering that the median spin period of the pulsars with He-WD companions is about $4 \mathrm{~ms}$ (a value derived from pulsars listed in the ATNF pulsar catalog 2 , Manchester et al. 2005), the spin period of PSR J1618-3921 appears to be

2 see http://www.atnf.csiro.au/research/pulsar/psrcat an outlier. However, Tauris et al. (2012) have pointed out half a dozen similar relatively slow spinning MSPs with apparent He-WD companions.

Because of the modest accuracy of the NRT timing for this low $\mathrm{S} / \mathrm{N}$ pulsar, we were unable to measure any post-Keplerian parameters from which we might have derived further constraints on the masses of the binary. We were likewise unable to measure a significant timing parallax, which is unsurprising given the large DM distance estimates for this pulsar (see Table 3). However, we attempted to measure the pulsar proper motion, since this is a key parameter for probing the formation process of eMSPs. As discussed in the following section, the different system formation scenarios predict varying initial kicks, so that the proper motion can be used to distinguish between them. A direct TEMPO2 fit did not lead to a significant proper motion measurement. We therefore produced a map of the $\chi^{2}$ of the timing residuals (see Fig. 3), with proper motions in right ascension $\mu_{\alpha}$ and declination $\mu_{\delta}$ fixed at values ranging from -20 to

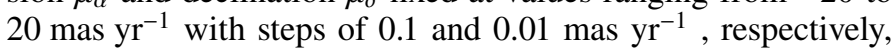




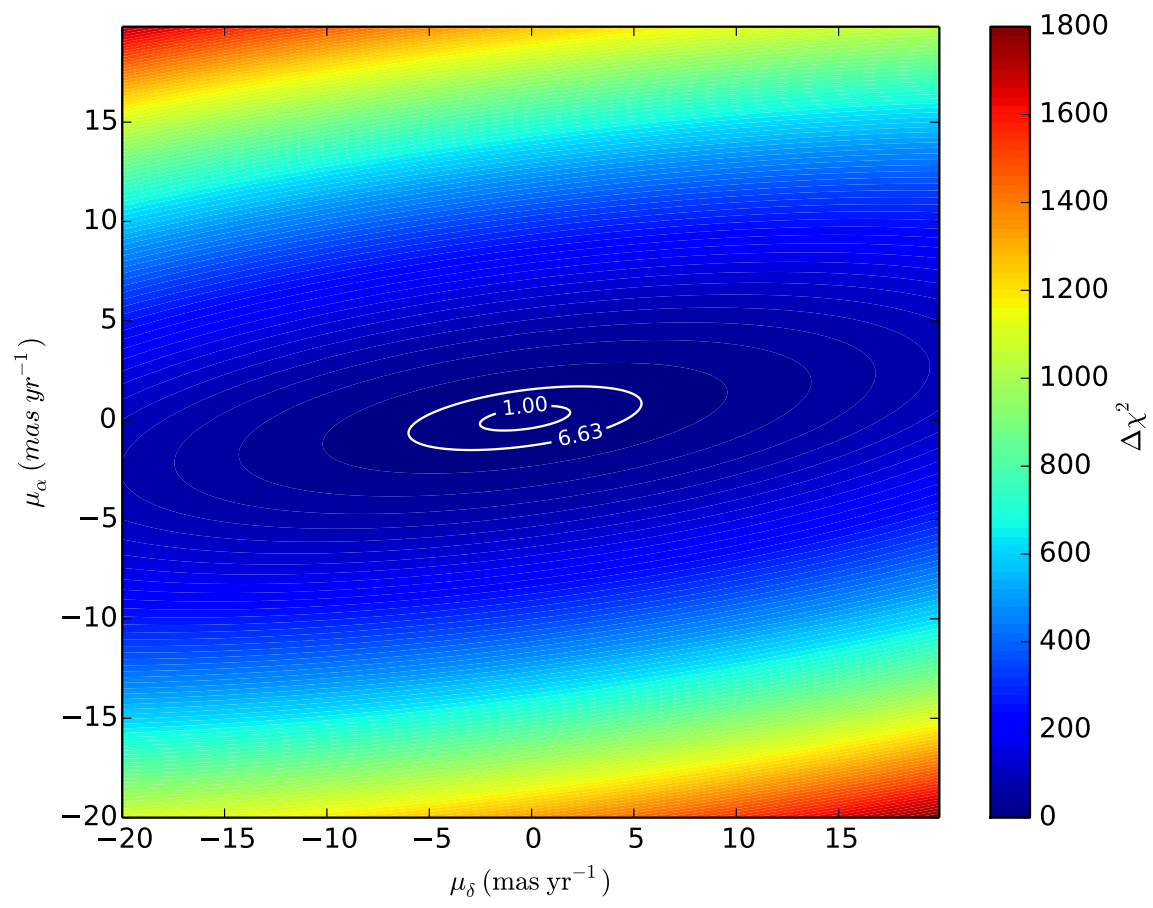

Fig. 3. Map of the $\chi^{2}$ variations as a function of the proper motions in right ascension and declination. Solid curves at $\Delta \chi^{2}=1.0$ and 6.63 correspond to uncertainties of $1 \sigma$ and $3 \sigma$, respectively. and fitting for the other parameters in Table 3 . The minimum

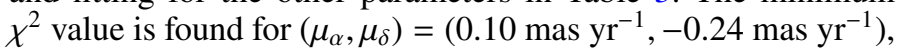
but the $1 \sigma$ contour makes it consistent with 0 . However, from the maximum transverse proper motion $\mu_{T}=\left(\mu_{\alpha}^{2}+\mu_{\delta}^{2}\right)^{1 / 2}$ along the $3 \sigma$ contour (corresponding to $\Delta \chi^{2}=6.63$ ), we were able to determine an upper limit on the transverse velocity of PSR J1618-3921 of about $80 \mathrm{~km} \mathrm{~s}^{-1}$, assuming the NE2001 model distance of $2.7 \mathrm{kpc}$. Assuming the YMW16 model distance of $5.5 \mathrm{kpc}$ leads to a $3 \sigma$ upper limit on the velocity of $160 \mathrm{~km} \mathrm{~s}^{-1}$. The two limits bracket the mean velocity of recycled pulsars of $\sim 90 \mathrm{~km} \mathrm{~s}^{-1}$ (Hobbs et al. 2005), suggesting that the transverse velocity of PSR J1618-3921 might be low or typical, but not high.

\section{Discussion}

The high characteristic age $(3.5 \mathrm{Gyr})$, relatively small surface magnetic field strength $\left(8.4 \times 10^{8} \mathrm{G}\right)$, and the low minimum companion mass $\left(0.17 M_{\odot}\right)$ of PSR J1618-3921 (see Table 3) are typical of MSPs formed by the recycling scenario (Tauris et al. 2012). In this scenario, the pulsar is spun-up by mass and angular momentum transfer from a companion star in a low-mass X-ray binary. Owing to a mass-transfer timescale that is much longer than the tidal-circularization timescale, the orbits of binary MSPs are highly circularized, except for systems that formed in a globular cluster, where the high stellar density may perturb the orbit. Considering that PSR J1618-3921 does not belong to a globular cluster, the eccentricity of 0.027 is abnormal. We now discuss various hypotheses to explain this high eccentricity.

Freire \& Tauris (2014) proposed the rotationally delayed, accretion-induced collapse (RD-AIC) of a super-Chandrasekhar mass WD. In this model, the binary system evolves from a system with a massive ONeMg WD accreting from a low-mass companion star in a CV-like binary. After the mass transfer ceases, the system is composed of two WDs, a super-massive $\mathrm{ONeMg}$ WD and a He WD. As a result of its rapid spin, the ONeMg WD was able to grow beyond the Chandrasekhar limit of $1.37 M_{\odot}$ for a non-rotating WD (Yoon \& Langer 2004, and references therein). The transition toward a neutron star-WD system occurs after the accretion phase when the ONeMg WD loses spin angular momentum and the centrifugal forces are no longer sufficient to sustain the stability of the WD. It implodes and forms a neutron star. In this collapse process, the sudden release of gravitational binding energy increases the orbital period and imposes an eccentricity on the system. The observed properties of the resulting system do follow the $\left(M_{W D}, P_{b}\right)$ relation (Tauris \& Savonije 1999). Monte Carlo simulations made by Freire \& Tauris (2014) revealed that to form a pulsar such as PSR J1618-3921 (with an orbital period of 22.7 days and an eccentricity of 0.027), a small kick of $w \sim 5 \mathrm{~km} \mathrm{~s}^{-1}$ is necessary. Such small kicks are indeed expected from the similar electron capture supernovae (Kitaura et al. 2006). In the simulations of Freire \& Tauris (2014), the masses of the $\mathrm{ONeMg}$ WD and its He WD companion were randomly chosen between $1.37-1.48 M_{\odot}$ and $0.26-0.28 M_{\odot}$, respectively. The RD-AIC model has the advantage to correctly account for some of the main observational and evolutionary characteristics of PSR J1618-3921 (and of eMSPs in general). In particular, i) the observed mass of the He WD companion fits the $\left(M_{\mathrm{WD}}, P_{b}\right)$-correlation for He WDs (Tauris \& Savonije 1999); ii) computations of the mass-transfer rate from its progenitor star (with its given orbital period) fall within the window where an ONeMg WD can accrete and grow in mass beyond the Chandrasekar limit for non-rotating WDs (Tauris et al. 2013); and iii) the predicted eccentricities fit the observed values. Nevertheless, the RD-AIC predicts low space velocities and MSP masses, which is in contradiction with the observation of at least one eMSP (PSR J1946+3417, Barr et al. 2017).

An alternative formation hypothesis was put forward by Antoniadis (2014), who demonstrated that the interaction of the binary with a circumbinary disk, fed by matter escaping the proto-WD during hydrogen shell flash episodes, may produce an eccentric system. For example, an interaction with a circumbinary disk with a typical mass of $\approx(1-9) \times 10^{-4} M_{\odot}$ and a lifetime of $10^{4}-10^{5} \mathrm{yr}$ is sufficient to produce an eccentric system with an eccentricity of $0.01-0.13$ and an orbital period of 
Table 3. Parameters for PSR J1618-3921 derived from the timing analysis.

\begin{tabular}{ll}
\hline \hline \multicolumn{1}{c}{ Timing and binary parameters } \\
\hline Right ascension, $\alpha(\mathrm{J} 2000)$ & $16: 18: 18.8248(3)$ \\
Declination, $\delta(\mathrm{J} 2000)$ & $-39: 21: 01.815(10)$ \\
Rotational period, $P(\mathrm{~ms})$ & $11.987308585310(22)$ \\
Period derivative, $\dot{P}\left(10^{-20}\right)$ & $5.408(18)$ \\
Dispersion measure, $\mathrm{DM}\left(\mathrm{cm}^{-3} \mathrm{pc}\right)$ & $117.965(11)$ \\
Orbital period, $P_{b}(\mathrm{~d})$ & $22.74559403(19)$ \\
Projected semi-major axis of orbit, $x(\mathrm{lt}-\mathrm{s})$ & $10.278300(5)$ \\
Epoch of periastron, $T_{0}(\mathrm{MJD})$ & $56012.21639(15)$ \\
Longitude of periastron, $\omega\left(^{\circ}\right)$ & $-6.717(3)$ \\
Orbital eccentricity, $e$ & $0.0274133(10)$ \\
Span of timing data $(\mathrm{MJD})$ & $54963.0-57869.1$ \\
Number of TOAs & 70 \\
Weighted residual rms $(\mu \mathrm{s})$ & 25.3 \\
Reduced $\chi^{2}$ value & 1.2 \\
\hline & \\
\hline Mass function, $f\left(M_{\odot}\right)$ & $0.00225347(9)$ \\
Minimum companion mass, $m_{\mathrm{c}}\left(M_{\odot}\right)$ & 0.1736 \\
Galactic longitude, $l\left(^{\circ}\right)$ & 340.72 \\
Galactic latitude, $b\left(^{\circ}\right)$ & 7.89 \\
Proper motion, $\mu_{T}\left(\mathrm{mas}\right.$ yr $\left.{ }^{-1}\right)$ & $<6.0$ \\
Characteristic age, $\tau\left(10^{9}\right.$ yr $)$ & 3.5 \\
Surface magnetic field strength, $B\left(10^{8} \mathrm{G}\right)$ & 8.14 \\
Magnetic field strength at the light cylinder, $B_{\mathrm{LC}}\left(10^{3} \mathrm{G}\right)$ & 4.4 \\
Spin-down luminosity, $\dot{E}\left(10^{33}\right.$ erg s $\left.{ }^{-1}\right)$ & 1.24 \\
Distance inferred from the NE2001 model, $d_{\mathrm{NE} 2001}(\mathrm{kpc})$ & 2.7 \\
Distance inferred from the $\mathrm{YMW16} \mathrm{model,} d_{\mathrm{YMw} 16}(\mathrm{kpc})$ & 5.5 \\
\hline
\end{tabular}

Notes. The reference epoch for the position and for the period is MJD 56000. We used the DE421 solar system ephemeris, and time units are in barycentric dynamic time (TDB). Numbers in parentheses represent $1 \sigma$ TEMPO2 uncertainties in the last digits quoted. The DM value was estimated independently from the other parameters (see Sect. 2). The pulsar mass used to calculate the minimum companion mass is $1.35 M_{\odot}$. See Lorimer \& Kramer (2004) for the calculation of $\tau, B, B_{\mathrm{LC}}$ and $\dot{E}$. Distance estimates were inferred from the NE2001 model of Cordes \& Lazio (2002) and the YMW16 model of Yao et al. (2017).

15-50 days. In this scenario, the eMSP would also follow the $\left(M_{W D}, P_{b}\right)$ relation. Although the input physics remains uncertain (e.g., Rafikov 2016), the clear advantage of this formation scenario is that it does not impose any limitations on pulsar mass, nor on the systemic velocity of the eMSP.

Jiang et al. (2015) proposed a model where a sudden mass loss that leads to the observed orbital eccentricity is caused by a phase transition in the pulsar interior due to quark deconfinement. This phase transition occurs when the core density of the neutron star reaches a critical density of $\sim 5 \rho_{0}$, where $\rho_{0}=$ $2.7 \times 10^{14} \mathrm{~g} \mathrm{~cm}^{-3}$ is the nuclear saturation density. This process of quark deconfinement is not well known and the involved physics remains uncertain.

Finally, a fourth possibility for producing eMSPs is a triple star interaction in which one star is kicked out of the system, thereby leaving a fossil eccentricity to the remaining binary. This scenario is believed to have formed PSR J1903+0327 (Champion et al. 2008; Freire et al. 2011). The unevolved, main-sequence G-type companion star in this system $\left(P_{b}=95\right.$ days and $e=$ $0.44)$ simply cannot have been responsible for the recycling of this MSP. However, as argued by Freire \& Tauris (2014) and Knispel et al. (2015), for the remaining five eMSPs, this triplestar process is too "chaotic" to produce quite identical observed properties for all systems, that is, low eccentricities $(e \lesssim 0.15)$, similar orbital periods $\left(P_{b} \simeq 22-32\right.$ days), and similar companion star masses $\left(M_{2} \simeq 0.20-0.30 M_{\odot}\right)$. Simulations of triple-star interactions producing PSR J1903+0327 indeed confirm this argument (Pijloo et al. 2012). Moreover, the exchange scenario would not explain why eMSPs fit the $\left(M_{W D}, P_{b}\right)$ law.

Better knowledge of the proper motion of all eMSPs is therefore an important tool to distinguish further between the formation scenarios for eMSPs. We need to collect more data in order to determine the proper motion of this pulsar more accurately. Early indications from our proper motion study seem to favor a low value and thus a weak natal kick at the birth of the neutron star.

We note, in closing, the discovery by Keane et al. (2018) of PSR J1421-4409, an MSP with an orbital period of 30-d and a "normal" eccentricity of about $10^{-5}$. This binary pulsar demonstrates that regardless of the mechanism that causes the anomalously high eccentricity of eMSPs, it does not affect all binary systems in this orbital period range.

\section{Conclusion and future work}

The analysis of eight years of NRT data enabled us to obtain a fully coherent timing solution for the first time and to confirm the anomalous eccentricity of PSR J1618-3921 ( $e=0.027$ ), which was first announced by Bailes (2010). This, the orbital period, and the mass function of the system make it a member of an emerging class of eccentric binary MSPs with similar orbital characteristics whose formation process is poorly understood. We place an upper limit on the proper motion of the pulsar. An 
actual measurement of the proper motion, which would provide us with crucial information about the system formation, was not possible with the considered dataset. Continued timing observations with the NUPPI should enable us to measure the proper motion of the pulsar in a few years. In parallel, optical and/or infrared observations of the binary system would be beneficial, as they would provide important information about the companion star, which from the timing analysis is expected to be a He WD. Further discoveries of similar systems exhibiting anomalously high eccentricities would also be useful for understanding the formation of these peculiar binaries.

Acknowledgements. The Nançay Radio Observatory is operated by the Paris Observatory, associated with the French Centre National de la Recherche Scientifique (CNRS). We acknowledge financial support from "Programme National de Cosmologie et Galaxies" (PNCG) of CNRS/INSU, France. PCCF gratefully acknowledges financial support by the European Research Council for the ERC Starting grant BEACON and continued support from the Max Planck Society under contract No. 279702.

\section{References}

Alpar, M. A., Cheng, A. F., Ruderman, M. A., \& Shaham, J. 1982, Nature, 300, 728

Antoniadis, J. 2014, ApJ, 797, L24

Antoniadis, J., Kaplan, D. L., Stovall, K., et al. 2016, ApJ, 830, 36

Bailes, M. 2010, New Astron. Rev., 54, 80

Barr, E. D., Champion, D. J., Kramer, M., et al. 2013, MNRAS, 435, 2234

Barr, E. D., Freire, P. C. C., Kramer, M., et al. 2017, MNRAS, 465, 1711
Bhattacharya, D., \& van den Heuvel, E. P. J. 1991, Phys. Rep., 203, 1

Camilo, F., Kerr, M., Ray, P. S., et al. 2015, ApJ, 810, 85

Champion, D. J., Ransom, S. M., Lazarus, P., et al. 2008, Science, 320, 1309

Cordes, J. M., \& Lazio, T. J. W. 2002, ArXiv e-prints [arXiv:astro-ph/0207156]

Deneva, J. S., Stovall, K., McLaughlin, M. A., et al. 2013, ApJ, 775, 51

Edwards, R. T., \& Bailes, M. 2001, ApJ, 553, 801

Freire, P. C. C., Bassa, C. G., Wex, N., et al. 2011, MNRAS, 412, 2763

Freire, P. C. C., \& Tauris, T. M. 2014, MNRAS, 438, L86

Hobbs, G., Lorimer, D. R., Lyne, A. G., \& Kramer, M. 2005, MNRAS, 360, 974

Hobbs, G., Edwards, R., \& Manchester, R. 2006, Chin. J. Astron. Astrophys. Supp., 6, 189

Hotan, A. W., van Straten, W., \& Manchester, R. N. 2004, PASA, 21, 302

Jiang, L., Li, X.-D., Dey, J., \& Dey, M. 2015, ApJ, 807, 41

Keane, E. F., Barr, E. D., Jameson, A., et al. 2018, MNRAS, 473, 116

Kitaura, F. S., Janka, H.-T., \& Hillebrandt, W. 2006, A\&A, 450, 345

Knispel, B., Lyne, A. G., Stappers, B. W., et al. 2015, ApJ, 806, 140

Lorimer, D. R., \& Kramer, M. 2004, Handbook of Pulsar Astronomy, eds. R. Ellis, J. Huchra, S. Kahn, G. Rieke, \& P. B. Stetson

Manchester, R. N., Hobbs, G. B., Teoh, A., \& Hobbs, M. 2005, AJ, 129, 1993

Phinney, E. S., \& Kulkarni, S. R. 1994, ARA\&A, 32, 591

Pijloo, J. T., Caputo, D. P., \& Portegies Zwart S. F. 2012, MNRAS, 424, 2914

Radhakrishnan, V., \& Srinivasan, G. 1982, Current Sci., 51, 1096

Rafikov, R. R. 2016, ApJ, 830, 8

Tauris, T. M., \& Savonije, G. J. 1999, A\&A, 350, 928

Tauris, T. M., \& van den Heuvel, E. P. J. 2006, in Compact Stellar X-ray Sources, eds. W. H. G. Lewin \& M. van der Klis, 623

Tauris, T. M., Langer, N., \& Kramer, M. 2012, MNRAS, 425, 1601

Tauris, T. M., Sanyal, D., Yoon, S.-C., \& Langer, N. 2013, A\&A, 558, A39

Yao, J. M., Manchester, R. N., \& Wang, N. 2017, ApJ, 835, 29

Yoon, S.-C., \& Langer, N. 2004, A\&A, 419, 623 